\title{
Epigenetic regulation of lung cancer cell proliferation and migration by the chromatin remodeling protein BRG1
}

\author{
Zilong $\mathrm{Li}^{1}$, Jun $\mathrm{Xia}^{2}$, Mingming Fang ${ }^{3,4}$ and Yong $\mathrm{Xu}^{1,4}$
}

\begin{abstract}
Malignant lung cancer cells are characterized by uncontrolled proliferation and migration. Aberrant lung cancer cell proliferation and migration are programmed by altered cancer transcriptome. The underlying epigenetic mechanism is unclear. Here we report that expression levels of BRG1, a chromatin remodeling protein, were significantly upregulated in human lung cancer biopsy specimens of higher malignancy grades compared to those of lower grades. Small interfering RNA mediated depletion or pharmaceutical inhibition of BRG1 suppressed proliferation and migration of lung cancer cells. BRG1 depletion or inhibition was paralleled by down-regulation of cyclin B1 (CCNB1) and latent TGF- $\beta$ binding protein 2 (LTBP2) in lung cancer cells. Further analysis revealed that BRG1 directly bound to the CCNB1 promoter to activate transcription in response to hypoxia stimulation by interacting with E2F1. On the other hand, BRG1 interacted with Sp1 to activate LTBP2 transcription. Mechanistically, BRG1 regulated CCNB1 and LTBP2 transcription by altering histone modifications on target promoters. Specifically, BRG1 recruited KDM3A, a histone H3K9 demethylase, to remove dimethyl H3K9 from target gene promoters thereby activating transcription. KDM3A knockdown achieved equivalent effects as BRG1 silencing by diminishing lung cancer proliferation and migration. Of interest, BRG1 directly activated KDM3A transcription by forming a complex with HIF-1a. In conclusion, our data unveil a novel epigenetic mechanism whereby malignant lung cancer cells acquired heightened ability to proliferate and migrate. Targeting BRG1 may yield effective interventional strategies against malignant lung cancers.
\end{abstract}

\section{Introduction}

Lung cancer represents one of the most deadly cancers in both industrialized and developing countries ${ }^{1,2}$. During lung cancer oncogenesis, normal epithelial cells acquire the ability of aggressive proliferation and migration contributing to tumor growth and dissemination ${ }^{3}$. Aberrant cancer cell proliferation and migration are regulated by a

\footnotetext{
Correspondence: Jun Xia (ricsummer@163.com) or Yong Xu (yjxu@njmu.edu. $\mathrm{cn})$

${ }^{1}$ Key Laboratory of Targeted Intervention of Cardiovascular Disease and Collaborative Innovation Center for Cardiovascular Translational Medicine, Department of Pathophysiology, Nanjing Medical University, Nanjing, China ${ }^{2}$ Department of Respiratory Medicine, The Affiliated Hospital of Nanjing University of Chinese Medicine, Jiangsu Province Hospital of Traditional Chinese Medicine, Nanjing, China

Full list of author information is available at the end of the article. These authors contributed equally: Zilong Li, Mingming Fang
}

myriad of signaling pathways, which converge in the nucleus to re-program the cellular transcriptome. Typically, genes that promote cell proliferation and/or migration are up-regulated in lung cancer cells compared to the normal cells. For instance, hypoxia, a characteristic feature of the tumor microenvironment, promotes lung cancer cell proliferation and migration by activating the expression of genes involved in cell cycling (e.g., cyclins), epithelial-to-mesenchymal transition (e.g., Twist), glucose metabolism (e.g., Glut1), cell survival and apoptosis (e.g., $\mathrm{Bcl} 2$ ), and angiogenesis (e.g., VEGF $)^{4}$. Hypoxia-inducible factor (HIF) family of transcription factors are considered the primary mediator of the cellular response to hypoxia in lung cancer cells although other transcription factors may also play critical roles ${ }^{5}$. The epigenetic mechanism whereby hypoxia skews lung cancer cell transcriptome to

\section{(c) The Author(s) 2019}

(cc) Open Access This article is licensed under a Creative Commons Attribution 4.0 International License, which permits use, sharing, adaptation, distribution and reproduction c. in any medium or format, as long as you give appropriate credit to the original author(s) and the source, provide a link to the Creative Commons license, and indicate if changes were made. The images or other third party material in this article are included in the article's Creative Commons license, unless indicated otherwise in a credit line to the material. If material is not included in the article's Creative Commons license and your intended use is not permitted by statutory regulation or exceeds the permitted use, you will need to obtain permission directly from the copyright holder. To view a copy of this license, visit http://creativecommons.org/licenses/by/4.0/. 
promote proliferation and migration is not completely understood.

Brahma related gene 1 (BRG1) is the catalytic component of the mammalian SWI/SNF chromatin remodeling complex intimately involved in transcriptional regulation $^{6}$. BRG1 characteristically functions within the confines of a multifactor epigenetic complex that include not only other subunits of the SWI/SNF proteins (e.g., BRG1associated factors or BAFs) but histone/DNA modifying enzymes and non-coding regulatory RNAs ${ }^{7}$. The compositions of the BRG1-containing complexes appear to be rather flexible so that BRG1 regulates transcription in a cell type and context-dependent manner. BRG1 is essential for embryogenesis as evidenced by the observation that germline deletion of BRG1 in mice causes developmental arrest and lethality ${ }^{8}$. Postnatal activation of BRG1, however, has been found to be associated with a host of human diseases including atherosclerosis ${ }^{9}$, pulmonary hypertension ${ }^{10}$, pathological hypertrophy ${ }^{11}$, alcoholic steatohepatitis ${ }^{12,13}$, and abdominal aortic aneurysm ${ }^{14}$.

The relationship between BRG1 and lung carcinogenesis remains controversial with evidence arguing both for and against BRG1 being a promoter of lung cancer development and progression. On the one hand, loss-offunction mutations of BRG1 have been frequently identified in lung cancer cells ${ }^{15}$. In addition, BRG1 inactivation is associated with increased lung cancer aggressiveness in humans ${ }^{16}$, which seems to suggest that BRG1 may function as a suppressor of lung oncogenesis. In contrast, loss of BRG1 sensitizes non-small cell lung cancer to chemotherapeutic drugs targeting either $\mathrm{CDK} 4 / 6^{17}$ or aurora kinase $\mathrm{A}^{18}$. In the present study, we investigated the mechanism whereby BRG1 regulates hypoxia-induced proliferation and migration of lung cancer cells. We report that BRG1 contributes to hypoxia-induced transcription of genes involved in cancer cell proliferation and migration by interacting with different sequence-specific transcription factors. BRG1 activates transcription in lung cancer cells by recruiting the demethylase KDM3A and by directly up-regulating KDM3A expression. Therefore, targeting BRG1 may yield effective interventional strategies against malignant lung cancers.

\section{Results}

Elevated BRG1 expression correlates with augmented lung cancer malignancy in humans

We first examined the expression levels of BRG1 in a small cohort of lung cancer biopsy specimens. Compared to those of low malignancies (grade I and grade II), highly malignant tumors (grade III and grade IV) exhibit much higher expression of BRG1 (SMARCA4, Fig. 1a). We also compared the expression of genes involved in cell proliferation and migration between the two groups. Whereas cyclin B1 (CCNB1, Fig. 1c), cell division cycle associated 2 (CDCA2, Fig. 1e), and latent TGF binding protein 2 (LTBP2, Fig. 1g) were up-regulated in malignant lung cancers, cyclin A1 (CCNA1, Fig. 1b), cyclin D1 (CCND1, Fig. 1d), and latent TGF binding protein 1 (LTBP1, Fig. 1f) were not significantly altered. We then exposed lung cancer cells (LLC) to the treatment of low oxygen tension (hypoxia), which is known to promote cancer cell proliferation and migration ${ }^{19,20}$. Hypoxia exposure stimulated BRG1 expression in a time course dependent manner at both mRNA (Fig. 1i) and protein (Fig. 1j) levels.

\section{BRG1 regulates lung cancer cell proliferation and migration}

To examine whether BRG1 could regulate hypoxiainduced proliferation and migration of lung cancer cells, the following experiments were performed. MTT assay and wound healing assay showed that exposure to $1 \% \mathrm{O} 2$ augmented proliferation and migration of LLC cells by $\sim 35$ and $110 \%$, respectively; over-expression of BRG1 further enhanced hypoxia-induced LLC proliferation (Fig. $1 \mathrm{k}$ ) and migration (Fig. 11). Of note, BRG1 overexpression by itself did not significantly alter proliferation (Fig. 1k) or migration (Fig. 1l) suggesting that the ability of BRG1 to regulate cancer cell behavior may require a specific pro-malignancy stimulus (e.g., hypoxia). On the contrary, depletion of BRG1 by siRNA abolished hypoxia-induced proliferation (Fig. $1 \mathrm{~m}$ ) and migration (Fig. 1n) of LLC cells.

BRG1 is essential for hypoxia-induced expression of cyclin B1 and latent TGF binding protein 2 in lung cancer cells

The observations that there was a concurrent upregulation of BRG1, CCNB1, and LTBP2 in high-grade human lung cancers combined with the well-established roles of CCNB1 and LTBP2 in cancer cell proliferation and migration prompted us to investigate whether BRG1 may contribute to hypoxia-induced LLC cell proliferation and migration by regulating CCNB1 and LTBP2 expression. As shown in Fig. 2a, b, over-expression of BRG1 augmented hypoxia-induced expression of CCNB1 and LTBP2. In contrast, depletion of BRG1 by two separate pairs of siRNAs dampened induction of CCNB1 and LTBP2 by hypoxia in LLC cells (Fig. 2c, d). In addition, treatment with a specific BRG1 inhibitor PFI-3 dosedependently suppressed hypoxia-induced expression of CCNB1 and LTBP2 (Fig. 2e, f).

\section{BRG1 regulates CCNB1 transcription in lung cancer cells}

We next investigated whether BRG1 directly regulates CCNB1 transcription. Reporter assay showed that BRG1 augmented activation of the CCNB1 promoter $(-1075)$ by hypoxia (Fig. 3a). When a series of deletions were introduced, BRG1 was able to activate the CCNB1 promoter 


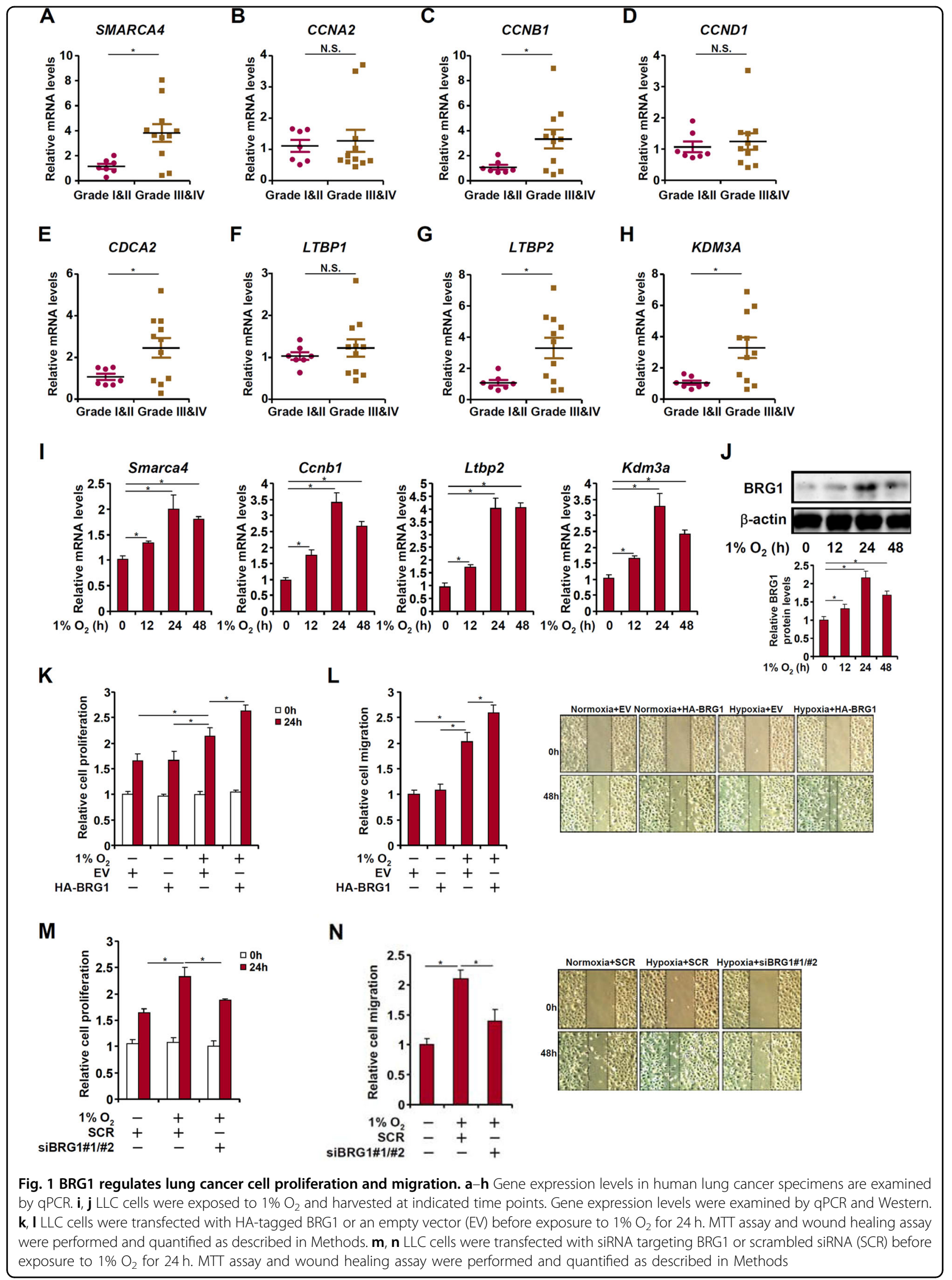


A

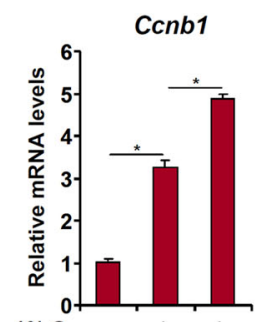
$\begin{array}{rrrrr}1 \% \mathrm{O}_{2} & - & + & + & 1 \% \mathrm{O}_{2} \\ \mathrm{EV} & + & + & - & \mathrm{EV} \\ \text { HA-BRG1 } & - & - & + & \text { HA-BRG } 1\end{array}$

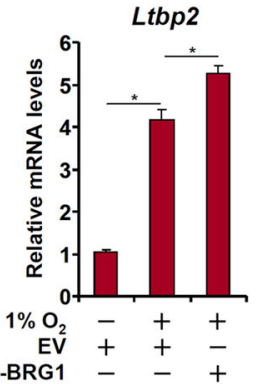

B

C

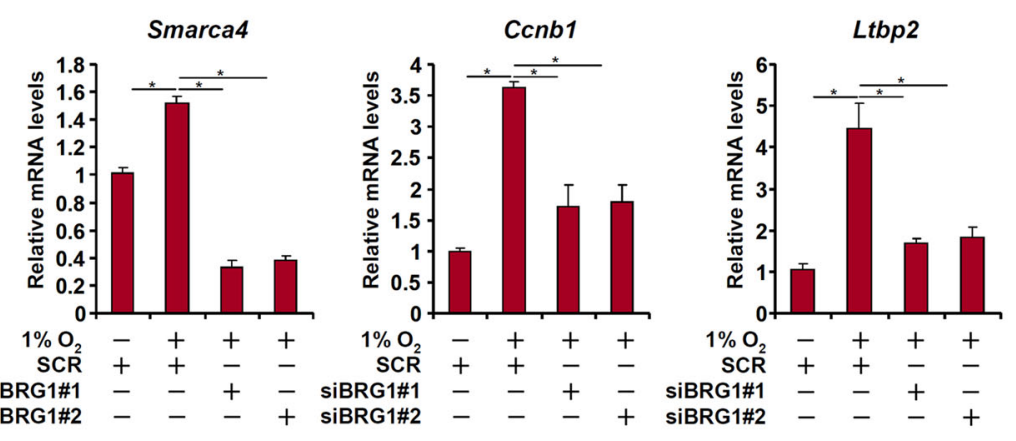

D
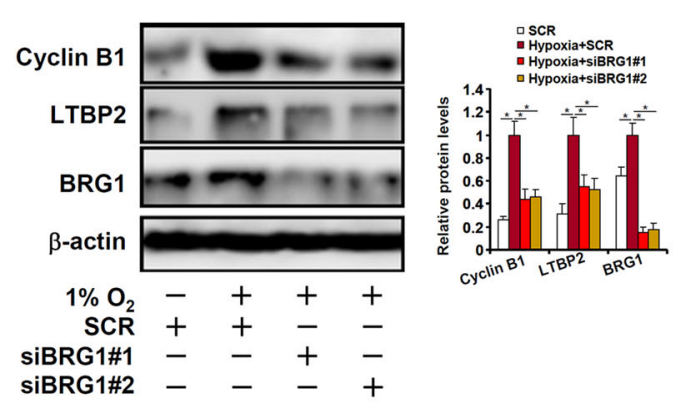

E

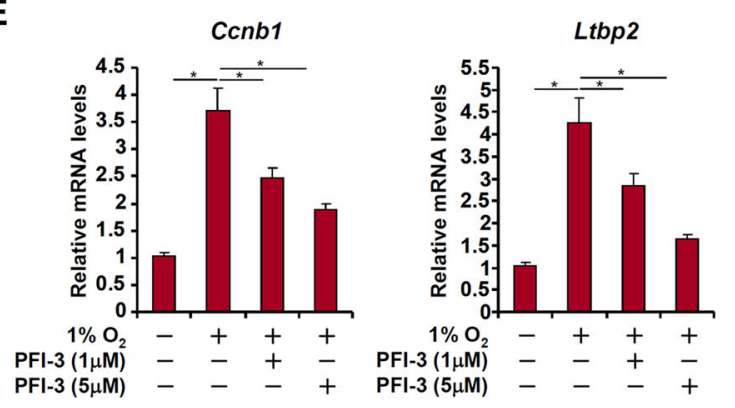

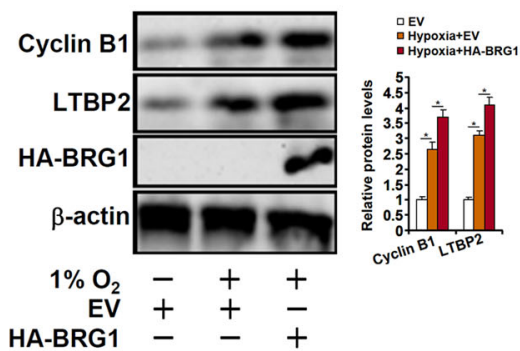

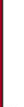

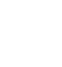


A

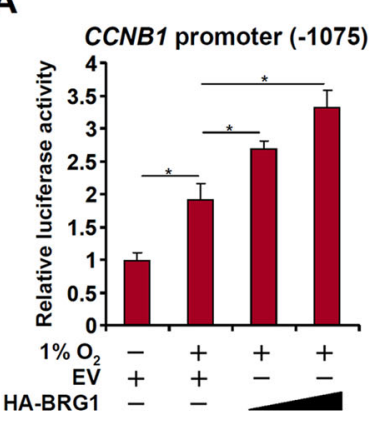

C
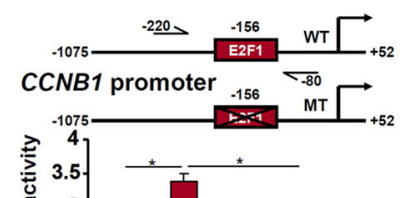

势 3.5

ฆ 2.5

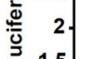

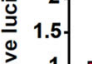

要 0.5 .

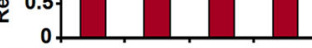

$1 \% \mathrm{O}_{2}$ AA-BRG 1

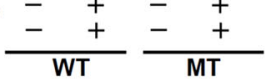

B

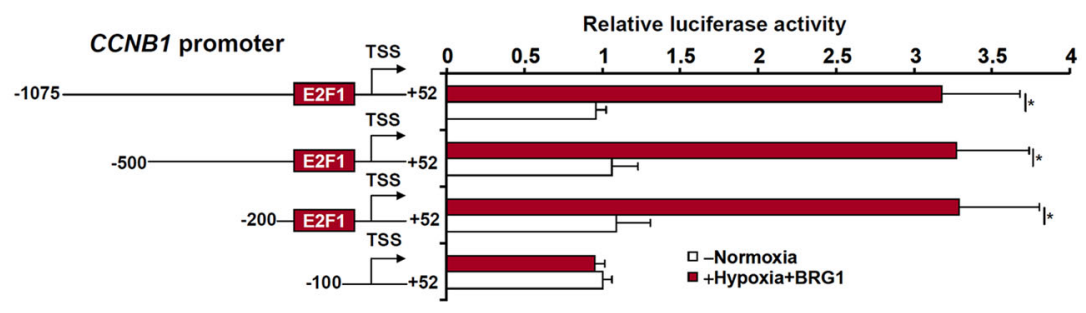

D

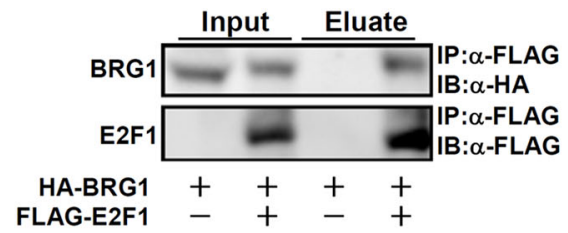

E
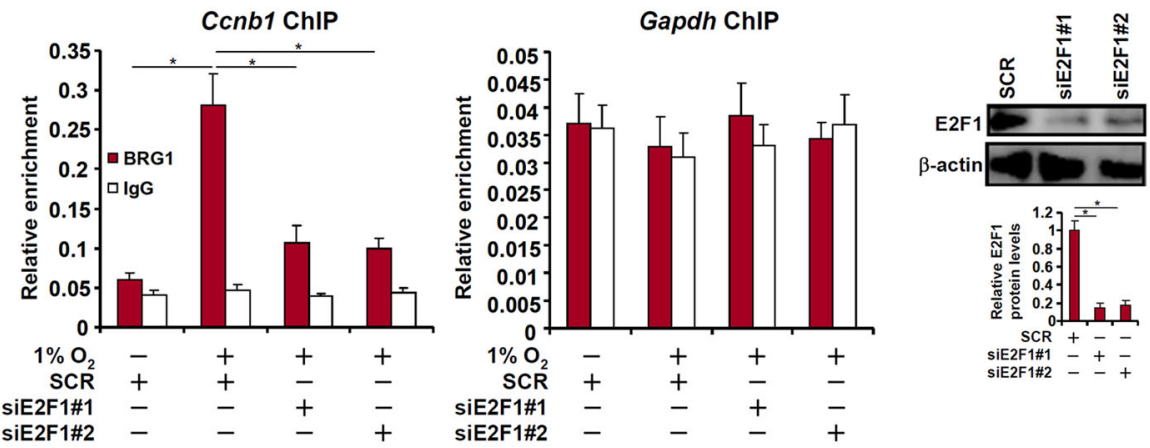

$\mathbf{F}$

Gapdh ChIP
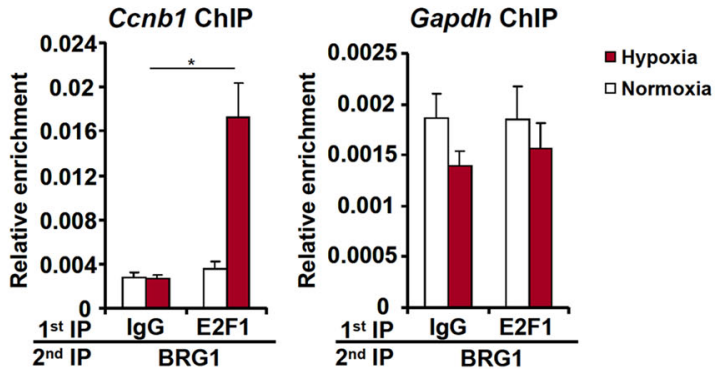

Fig. 3 (See legend on next page.) 
(see figure on previous page)

Fig. 3 BRG1 regulates CCNB1 transcription in lung cancer cells. a A CCNB1 promoter-luciferase construct was transfected into LLC cells with or without BRG1 followed by exposure to $1 \% \mathrm{O}_{2}$ for $24 \mathrm{~h}$. Luciferase activities were normalized by both protein concentration and GFP fluorescence. b CCNB1 promoter-luciferase constructs of different lengths were transfected into LLC cells with or without BRG1 followed by exposure to $1 \% \mathrm{O}_{2}$ for $24 \mathrm{~h}$. Luciferase activities were normalized by both protein concentration and GFP fluorescence. $\mathbf{c}$ Wild type and mutant CCNB1 promoter-luciferase constructs were transfected into LLC cells with or without BRG1 followed by exposure to $1 \% \mathrm{O}_{2}$ for $24 \mathrm{~h}$. Luciferase activities were normalized by both protein concentration and GFP fluorescence. d HA-tagged BRG1 and FLAG-tagged E2F1 were transfected into HEK293 cells. Co-

immunoprecipitation was performed with indicated antibodies. e LLC cells were transfected with siRNA targeting E2F1 or SCR before exposure to 1\% $\mathrm{O}_{2}$ for $24 \mathrm{~h}$. ChIP assays were performed with anti-BRG1 or lgG. Inset, knockdown efficiency of E2F1. $\mathbf{f} L L C$ cells were exposed to $1 \% \mathrm{O}_{2}$ and harvested $24 \mathrm{~h}$ later. Re-ChIP assay was performed with indicated antibodies

until the deletion went beyond -100 (Fig. 3b). Similarly, mutagenesis of a conserved E2F1 site within the CCNB1 promoter abrogated its activation by BRG1 (Fig. 3c), suggesting that BRG1 might rely on E2F1 to activate CCNB1 transcription. Indeed, co-immunoprecipitation showed that BRG1 interacted with E2F1 in cells (Fig. 3d). Further, BRG1 was recruited to the CCNB1 promoter in LLC cells exposed to low oxygen in an E2F1-dependent manner (Fig. 3e). Hypoxia treatment promoted the formation of a BRG1-E2F1 complex on the CCNB1 promoter (Fig. 3f). Combined, these data support a role for BRG1 in regulating hypoxia-induced CCNB1 transcription by interacting with E2F1.

\section{BRG1 regulates LTBP2 transcription in lung cancer cells}

We next explored the mechanism whereby BRG1 regulates LTBP2 transcription in LLC cells in response to hypoxia stimulation. BRG1 over-expression enhanced the trans-activation of the LTBP2 promoter in hypoxiatreated LLC cells only when a conserved Sp1 site was left intact (Fig. 4a), suggesting that Sp1 might be necessary for recruiting BRG1 to the LTBP2 promoter. The interaction between $\mathrm{Sp} 1$ and BRG1 was confirmed by coimmunoprecipitation assay (Fig. 4b). Further, hypoxia treatment enhanced the interaction between BRG1 and Sp1 on the LTBP2 promoter but not the GAPDH promoter (Fig. 4c). Sp1 silencing by siRNA (Fig. 4d) or inhibition by mithramycin (Fig. 4e) blocked BRG1 recruitment to the LTBP2 promoter. Collectively, these data are consistent with the model wherein BRG1 contributes to hypoxia-induced LTBP2 transcription by interacting with Sp1.

\section{BRG1 recruits KDM3A to regulate transcription}

We performed a series of ChIP assays to evaluate the epigenetic mechanism whereby BRG1 regulates the transcription of CCNB1 and LTBP2 in LLC cells. Hypoxia stimulation promoted the accumulation of acetylated histone H3 (Fig. 5a) and trimethylated H3K4 (Fig. 5b) on the proximal CCNB1 and LTBP2 promoters; inhibition of BRG1 by PFI- 3 antagonized the enrichment of acetyl H3 and trimethyl H3K4. On the other hand, there was a decrease in dimethyl H3K9 levels on the proximal CCNB1 and LTBP2 promoters, which was abrogated by BRG1 inhibition (Fig. 5c). Concomitantly, hypoxia treatment resulted in increased recruitment of KDM3A, a demethylase specialized in removing dimethyl H3K9, whereas BRG1 inhibition blocked KDM3A recruitment (Fig. 5d). Co-immunoprecipitation confirmed that BRG1 formed a complex with KDM3A in cells (Fig. 5e). More important, Re-ChIP assay demonstrated that stronger BRG1KDM3A interaction could be detected on the CCNB1 promoter and the LTBP2 promoter following hypoxia stimulation in LLC (Fig. 5f). In addition, KDM3A also contributed to hypoxia-induced CCNB1 and LTBP2 expression in LLC cells (Fig. 5g, h). Functionally, KDM3A appeared to be equivalent to BRG1 in promoting lung cancer cell proliferation (Fig. 5i) and migration (Fig. 5j).

\section{BRG1 directly activates KDM3A transcription}

Previously it has been shown that KDM3A transcription is hypoxia inducible via a conserved HIF- $1 \alpha$ site within its proximal promoter ${ }^{21}$. Of note, KDM3A levels were elevated in malignant types of lung cancers paralleling BRG1 up-regulation (Fig. 1h). We therefore sought to determine whether BRG1 might play a role in hypoxia-induced KDM3A transcription. BRG1 over-expression enhanced KDM3A induction by hypoxia (Fig. 6a, b). Conversely, BRG1 depletion (Fig. 6c, d) or BRG1 inhibition (Fig. 6e, f) attenuated KDM3A induction by hypoxia, suggesting that BRG1 might directly control KDM3A levels in LLC cells. To further verify the role of BRG1 in hypoxia-induced KDM3A transcription, a wild-type KDM3A promoterluciferase construct and a KDM3A promoter construct harboring hypoxia response element (HRE) mutation were transfected into LLC cells. As expected, hypoxia increased the WT KDM3A promoter activity; BRG1 overexpression further enhanced the KDM3A promoter activity. Neither hypoxia nor BRG1 over-expression impacted the MT KDM3A promoter activity (Fig. 6g). ChIP assay confirmed that BRG1 bound to the KDM3A promoter region containing HRE in response to hypoxia (Fig. 6h). The interaction between HIF-1 $\alpha$ and BRG1 was further confirmed by co-immunoprecipitation (Fig. 6i) 
A

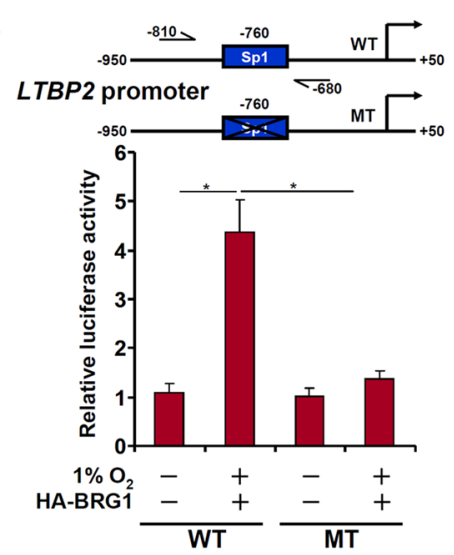

B

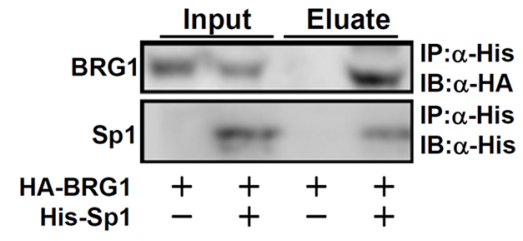

C
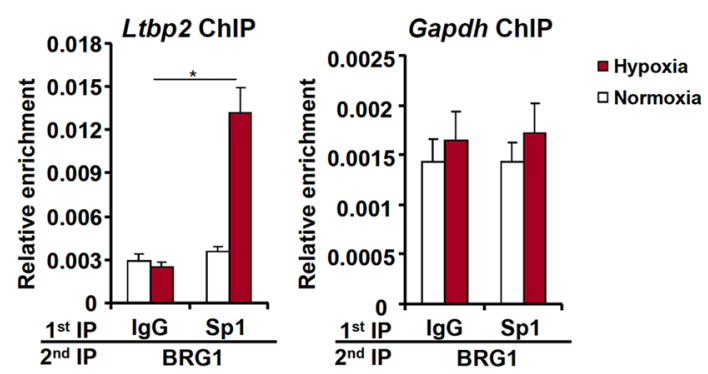

D
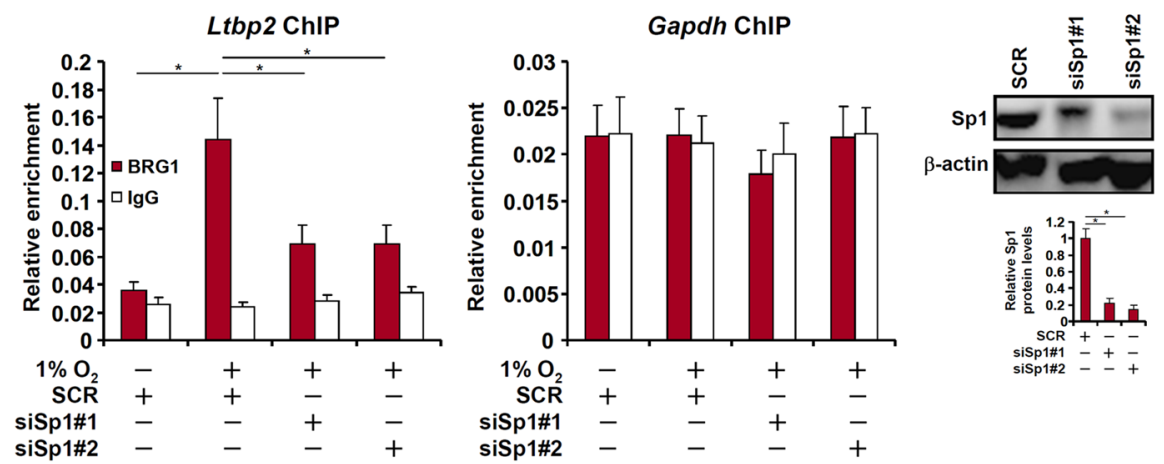

E

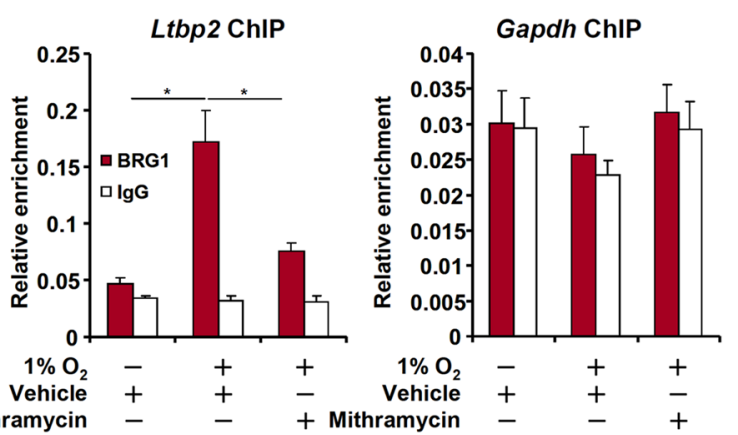

Fig. 4 BRG1 regulates LTBP2 transcription in lung cancer cells. a Wild type and mutant CCNB1 promoter-luciferase constructs were transfected into LLC cells with or without BRG1 followed by exposure to $1 \% \mathrm{O}_{2}$ for $24 \mathrm{~h}$. Luciferase activities were normalized by both protein concentration and GFP fluorescence. $\mathbf{b}$ HA-tagged BRG1 and His-tagged Sp1 were transfected into HEK293 cells. Co-immunoprecipitation was performed with indicated antibodies. c LLC cells were exposed to $1 \% \mathrm{O}_{2}$ and harvested $24 \mathrm{~h}$ later. Re-ChIP assay was performed with indicated antibodies. d LLC cells were transfected with siRNA targeting Sp1 or SCR before exposure to $1 \% \mathrm{O}_{2}$ for $24 \mathrm{~h}$. ChIP assays were perormed with anti-BRG1 or lgG. Inset, knockdown efficiency of Sp1. e LLC cells were treated with or without mithramycin before exposure to $1 \% \mathrm{O}_{2}$ for $24 \mathrm{~h}$. ChIP assays were perormed with antiBRG1 or $\operatorname{lgG}$ 
A

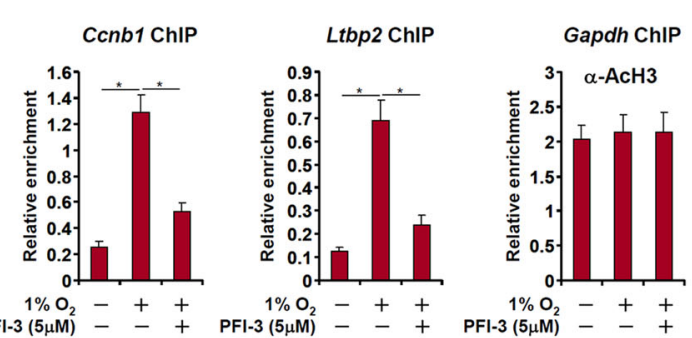

B

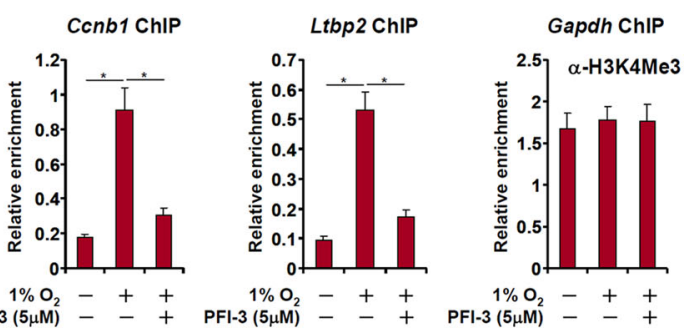

C

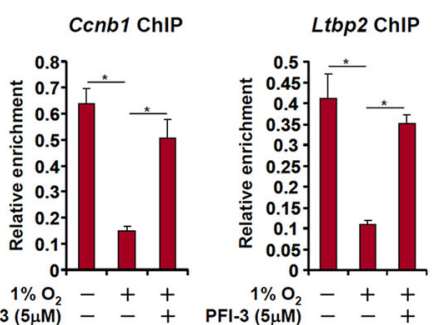

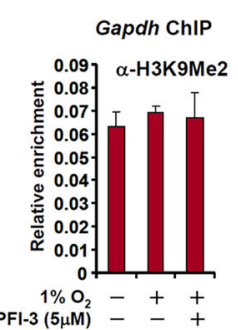

D
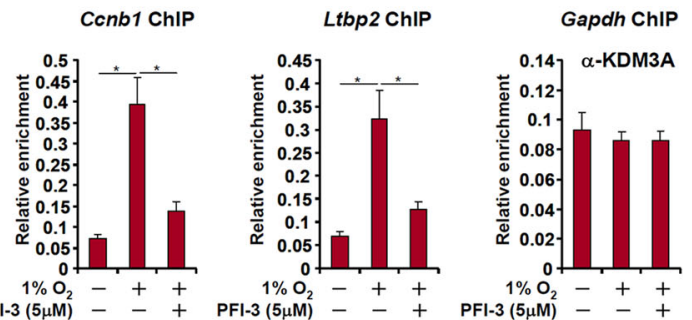

E
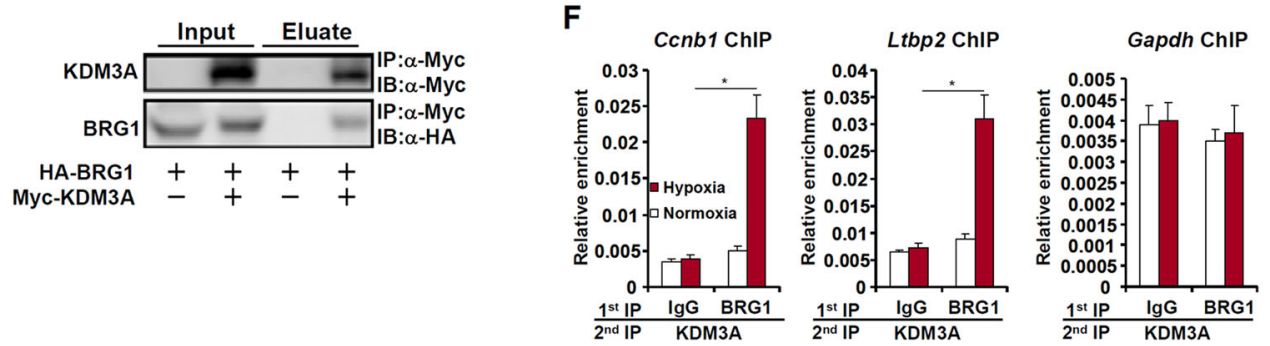

$\mathbf{G}$
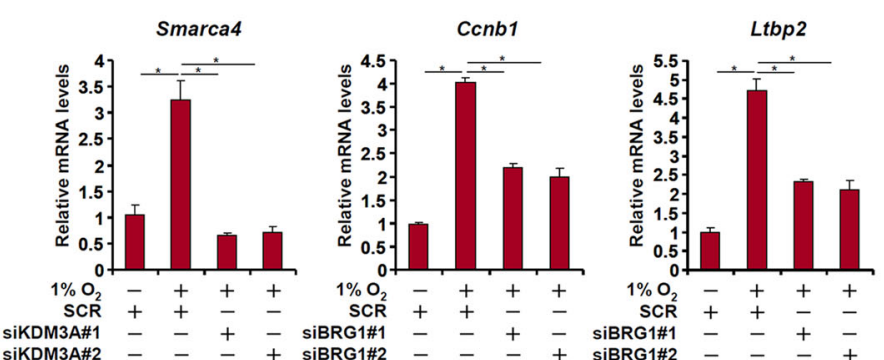

H
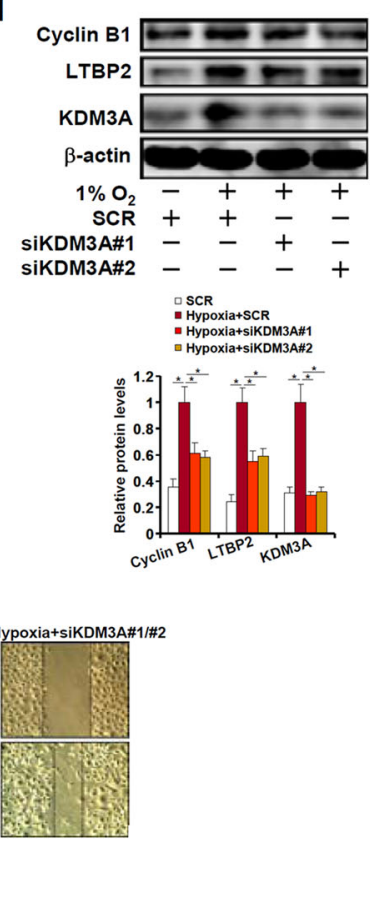

I

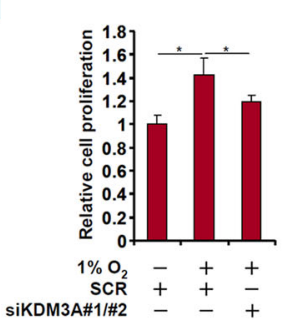

J

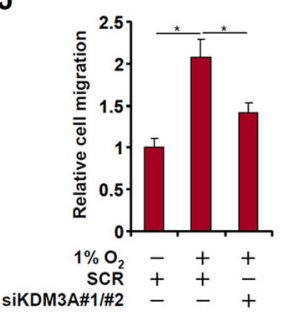

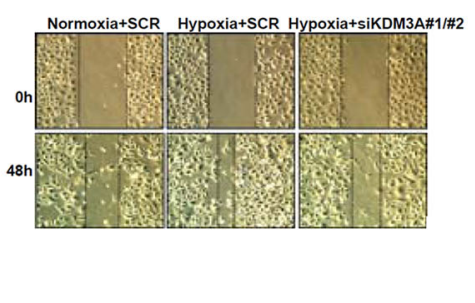

Fig. 5 BRG1 recruits KDM3A to regulate transcription. a-d LLC cells were treated with a BRG1 inhibitor and exposed to $1 \% \mathrm{O}_{2}$ for $24 \mathrm{~h}$. ChIP assays were performed with indicated antibodies. e HA-tagged BRG1 and Myc-tagged KDM3A were transfected into HEK293 cells. Coimmunoprecipitation was performed with indicated antibodies. $\mathbf{f}$ LLC cells were exposed to $1 \% \mathrm{O}_{2}$ and harvested $24 \mathrm{~h}$ later. Re-ChIP assay was performed with indicated antibodies. $\mathbf{g}$ - $\mathbf{j}$ LLC cells were transfected with siRNA targeting KDM3A or SCR before exposure to $1 \% \mathrm{O}_{2}$ for $24 \mathrm{~h}$. Gene expression levels were examined by qPCR and Western. MTT assay and wound healing assay were performed and quantified as described in Methods 


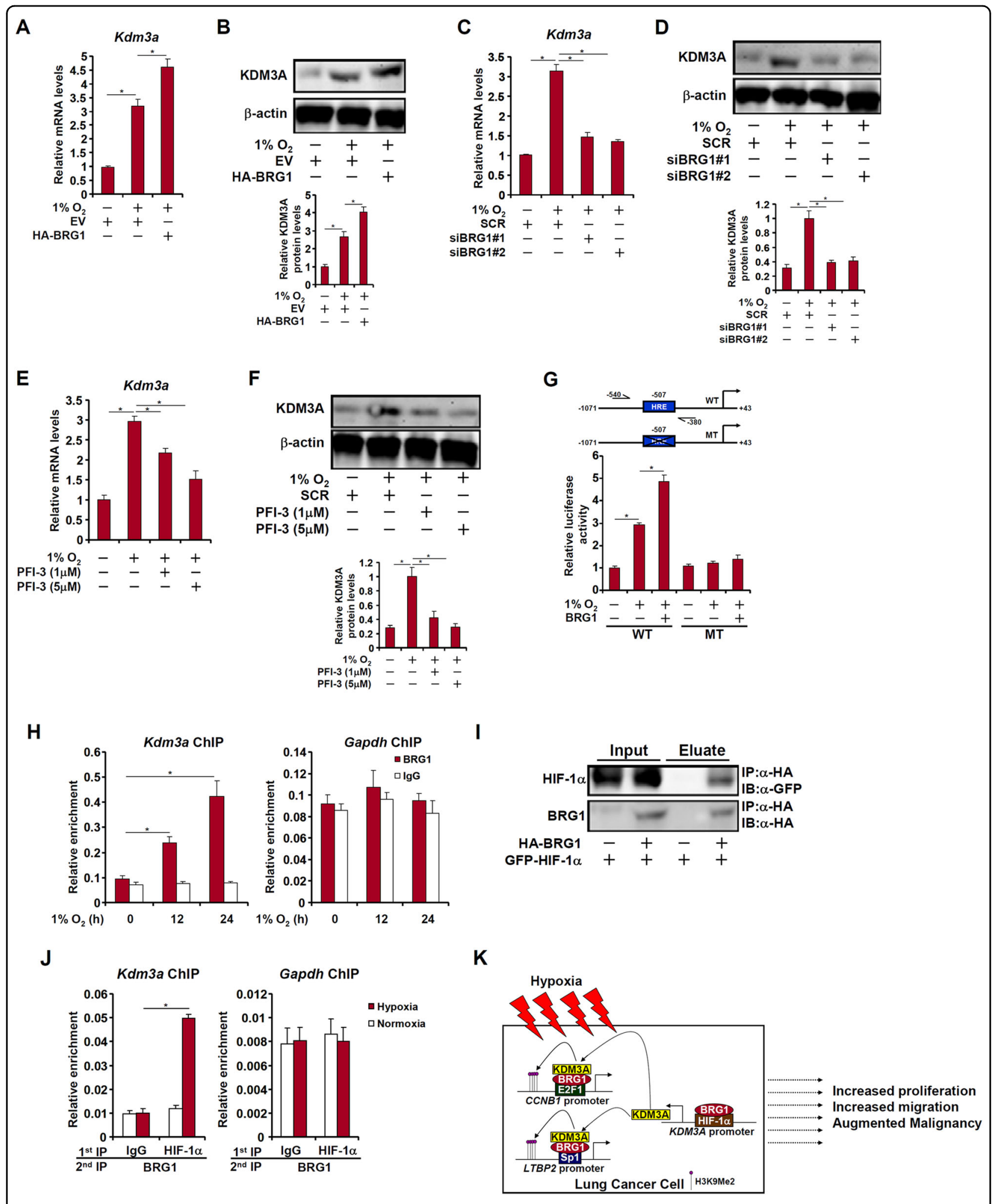

Fig. 6 (See legend on next page.) 
(see figure on previous page)

Fig. 6 BRG1 directly activates KDM3A transcription. $\mathbf{a}$, b LLC cells were transfected with HA-tagged BRG1 or an empty vector (EV) before exposure to $1 \% \mathrm{O}_{2}$ for $24 \mathrm{~h}$. Gene expression levels were examined by qPCR and Western. $\mathbf{c}$, $\mathbf{d}$ LLC cells were transfected with siRNA targeting BRG1 or SCR before exposure to $1 \% \mathrm{O}_{2}$ for $24 \mathrm{~h}$. Gene expression levels were examined by qPCR and Western. e, $\mathbf{f} L L C$ cells were treated with a BRG1 inhibitor and exposed to $1 \% \mathrm{O}_{2}$. Gene expression levels were examined by qPCR and Western. $\mathbf{g}$ Wild type and mutant KDM3A promoter-luciferase constructs were transfected into LLC cells with or without BRG1 followed by exposure to $1 \% \mathrm{O}_{2}$ for $24 \mathrm{~h}$. Luciferase activities were normalized by both protein concentration and GFP fluorescence. $\mathbf{h}$ LLC cells were exposed to $1 \% \mathrm{O}_{2}$ and harvested at indicated time points. ChIP assays were performed with anti-BRG1. i HA-tagged BRG1 and GFP-tagged HIF-1a were transfected into HEK293 cells. Co-immunoprecipitation was performed with indicated antibodies. $\mathbf{j}$ LLC cells were exposed to $1 \% \mathrm{O}_{2}$ and harvested $24 \mathrm{~h}$ later. Re-ChIP assay was performed with indicated antibodies. $\mathbf{k} \mathrm{A}$ schematic model

and Re-ChIP (Fig. 6j) assays. Taken together, these data demonstrate that BRG1 directly activates KDM3A transcription by interacting with HIF- $1 \alpha$.

\section{Discussion}

The epigenetic machinery is an integral part of the regulatory network that controls a wide range of pathological processes during cancer oncogenesis and metastasis including proliferation, migration, invasion, angiogenesis, metabolic reprogramming, immune evasion, and resistance to chemotherapy ${ }^{22}$. The effort to investigate the epigenetic mechanism underlying this heinous disease with the ultimate goal of identifying potential therapeutic targets in the past decade has grown exponentially. At the same time, our knowledge and understanding with regard to the epigenetic regulation of lung carcinogenesis has been expanded tremendously especially with the emergence of cancer epigenomics ${ }^{23}$. Here we present evidence to show that expression levels of BRG1, a chromatin remodeling protein, are elevated in human lung cancers as they become more aggressive paralleling the up-regulation of genes involved in cell proliferation (CCNB1) and migration (LTBP2). Further, BRG1 regulates pro-proliferative and pro-migratory transcription in lung cancer cells by interacting with different sequence-specific transcription factors. More important, BRG1 recruits a histone H3K9 demethylase KDM3A to alter the chromatin structure surrounding the target promoters. Finally, BRG1 directly controls the availability of KDM3A by cooperating with HIF- $1 \alpha$ to activate KDM3A transcription itself (Fig. 6k). Our data are consistent with recently published reports, which suggest that BRG1 confers growth and metastatic advantages to lung cancers ${ }^{17,18,24}$. Therefore, targeting BRG1 appears to be a viable strategy in the treatment of certain malignant forms of lung cancers.

BRG1 relies on its interactions with sequence-specific transcription factors to become integrated into the transcriptional network that programs cancer malignancy. Here we show that BRG1 interacts with E2F1 to regulate CCNB1 transcription and with Sp1 to regulate LTBP2 transcription. It has been previously suggested that BRG1 primarily functions as a co-repressor for E2F1 in the transcriptional regulation of pro-apoptotic genes such as $\mathrm{p} 14^{\mathrm{ARF}}$ and $\mathrm{p} 73^{25}$. However, it has been proposed that BRG1 can function either as a co-activator or a corepressor depending on the specific cell types and cues $^{26,27}$. It is not clear at this point whether the ability to potentiate E2F1-mediated CCNB1 transcription reflects the norm or an exception of the mode of action for BRG1 as an E2F1 co-factor. On the other hand, strong evidence suggests that BRG1 is a key co-activator for Sp1 in the regulation of oncogenesis ${ }^{28-30}$. An open question is whether interactions between BRG1 and factors other than E2F1/Sp1 may contribute to the regulation of lung cancer cell proliferation and migration by BRG1 because we suspect that such complex processes as cell proliferation and migration would depend on the expression of two, instead of a group of, genes (CCNB1 and LTBP2). Clearly, deciphering the BRG1 interactome with ChIP-seq will bring further insights to the understanding of lung cancer oncogenesis.

HIF- $1 \alpha$ is the master regulator of cellular response to hypoxia typical to a pro-proliferative and pro-migratory tumor microenvironment. Here we show that BRG1 collaborates with HIF-1 $\alpha$ to activate KDM3A transcription. Several known HIF-1 $\alpha$ target genes are sensitive to the alteration of BRG1 status although the mechanism is not invariably attributable to an interaction between BRG1 and HIF-1 $\alpha$. Kenneth et al. first reported that BRG1, along with other components of the SWI/SNF complex, is both sufficient and necessary for HIF-1 $\alpha$ dependent transcription as assayed by a hypoxia response element (HRE) reporter $^{31}$. Sena et al. have provided additional evidence to show that transcription of a subset of HIF-1a genes relies on BRG1 and/or BRM, the alternative ATPase subunit of the SWI/SNF complex, in hepatocellular carcinoma (HCC) cells ${ }^{32}$. Chen et al. have shown that BRG1 and BRM contributes to hypoxia-induced expression of adhesion molecules in vascular endothelial cells ${ }^{10}$. These findings allude to the possibility that BRG1 may be a de novo co-factor for HIF- $1 \alpha$ in modulating the hypoxia response. Several pieces are missing from the jigsaw puzzle of BRG1-mediated regulation of HIF- $1 \alpha$ activity. First, the interaction between BRG1 and HIF- $1 \alpha$ has yet to be fine-mapped. Second, how BRG1 contributes to HIF- 
$1 \alpha$ dependent transcription on a genomewide scale has not been determined. Finally, the relevance of this BRG1HIF-1a interplay in lung cancer oncogenesis has not been verified in model animals and in patients. These issues certainly deserve further investigation.

Mounting evidence points to a role for BRG1 in recruiting various histone and DNA modifying enzymes to target promoters to regulate transcription. We report here that BRG1 recruits KDM3A to activate CCNB1 and LTBP2 transcription in lung cancer cells. In addition, KDM3A is elevated in malignant type of human lung cancers and plays an essential role in hypoxia-induced lung cancer cell proliferation and migration. This is in keeping with recent findings that KDM3A promotes oncogenesis of colorectal cancer ${ }^{33}$, prostate cancer ${ }^{34}$, breast cancer ${ }^{35}$, and ovarian cancer ${ }^{36}$. Of interest, BRG1 is necessary for hypoxia-induced KDM3A transcription. Thus, BRG1 controls the KDM3A dynamics by not only modulating its physical association with target promoters but regulating its bioavailability. It would be of great interest to determine whether this BRG1-KDM3A interplay extends beyond the regulation of CCNB1 and LTBP2 transcription and how it contributes to lung cancer oncogenesis in vivo.

In summary, our data as summarized here point to a central role for BRG1 in the epigenetic regulation of lung cancer cell proliferation and migration in vitro. At least two different types of small-molecule BRG1 inhibitors are currently available ${ }^{37}$. Therefore, our data provide renewed rationale for using these chemicals and for exploring novel BRG1-targeting chemicals in clinical trials.

\section{Materials and methods}

\section{Human lung cancer samples}

All human studies were reviewed and approved by the intramural Nanjing Medical University Committee on Ethical Conduct of Studies with Human Subjects. Lung cancer tissues were collected, under informed consent, from surgical resection specimens of patients who had not undergone radiotherapy or chemotherapy in the Affiliated Hospital of Nantong University. Diagnoses of all cases were confirmed by histological examination. Tumor differentiation was graded by the Edmondson grading system. Samples were processed essentially as previously described $^{38}$. Basic patient information is summarized in supplementary Table I.

\section{Cell culture}

The murine lung carcinoma cells (LLC) were authenticated by the Chinese Academy of Sciences Type Culture Collection Cell Bank and were maintained in DMEM (Invitrogen) as previously described ${ }^{39}$. HEK293 cells were purchased from Invitrogen and maintained in DMEM. The cells were re-authenticated using a fingerprint method every 6 months in the laboratory. The last time the cells were authenticated was November 2018. Where indicated, hypoxia $\left(1 \% \mathrm{O}_{2}\right)$ was achieved by a mixture of ultra-high purity gases $\left(5 \% \mathrm{CO}_{2}, 10 \% \mathrm{H}_{2}, 85 \% \mathrm{~N}_{2}\right)$ in a $37^{\circ} \mathrm{C}$ incubator (Thermo Fisher).

\section{Plasmids, transient transfection, and reporter assay}

HA-tagged BRG1, FLAG-tagged E2F1, His-tagged Sp1, GFP-tagged HIF-1 $\alpha$, Myc-tagged KDM3A, CCNB1 promoter-luciferase constructs, and KDM3A promoterluciferase constructs have been previously described ${ }^{21,40-43}$. LTBP2 promoter-luciferase construct was made by amplifying $\sim 1 \mathrm{~kb}$ of genomic DNA spanning the proximal LTBP2 promoter $(-950 /+50)$ and ligating the amplicon into the pGL3 vector (Promega). Mutation constructs were made using the Quickchange Mutagenesis Kit (Agilent). Cells were harvested $48 \mathrm{~h}$ after transfection and reporter activity was measured using a luciferase reporter assay system (Promega) as previously described ${ }^{44-46}$. Briefly, cells were plated in 12-well culture dishes ( $\sim 60,000$ cells/well). The next day, equal amounts $(0.1 \mu \mathrm{g})$ of reporter construct and effector construct were transfected into each well. DNA content was normalized by the addition of an empty vector (pcDNA3). For monitoring transfection efficiency and for normalizing luciferase activity, $0.02 \mu \mathrm{g}$ of GFP construct was transfected into each well. Luciferase activities were normalized by both protein concentration and GFP fluorescence. Data are expressed as relative luciferase unit compared to the control group arbitrarily set as 1 .

\section{Protein extraction, immunoprecipitation, and western blot}

Whole-cell lysates were obtained by re-suspending cell pellets in RIPA buffer ( $50 \mathrm{mM}$ Tris pH 7.4, $150 \mathrm{mM} \mathrm{NaCl}$, $1 \%$ Triton X-100) with freshly added protease inhibitor (Roche) as previously described ${ }^{47,48}$. Specific antibodies or pre-immune IgGs (P.I.I.) were added to and incubated with cell lysates overnight before being absorbed by Protein A/G-plus Agarose beads (Santa Cruz). Precipitated immune complex was released by boiling with 1X SDS electrophoresis sample buffer. Western blot analyses were performed with anti-BRG1 (Santa Cruz, sc10768), anti-Cyclin B1 (Proteintech, 55004-1), antiLTBP2 (Sigma, HPA003415), anti-KDM3A (Proteintech, 12835-1), anti-HA (Sigma, H3663), anti-FLAG (Sigma, F3165), anti-His (Invitrogen, MA1-21315), anti-GFP (Proteintech, 50430-2), anti-Myc (Santa Cruz, sc-40), and anti- $\beta$-actin (Sigma, A2228) antibodies. All experiments were repeated three times.

\section{RNA isolation and real-time PCR}

RNA was extracted with the RNeasy RNA isolation kit (Qiagen) as previously described ${ }^{49,50}$. Reverse transcriptase reactions were performed using a SuperScript 
First-strand Synthesis System (Invitrogen). Real-time PCR reactions were performed on an ABI Prism 7500 system with the following primers: human BRG1, 5' -AGTGCTG

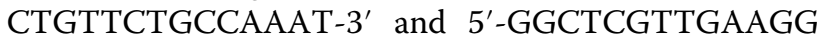
TTTTCAG-3'; human CCNA2, 5'-TACCTGGACCCA GAAAACCA- $3^{\prime}$ and $5^{\prime}$-CACTCACTGGCTTTTCATCT TCT-3'; human CCNB1, 5'-TGTGGATGCAGAAG ATGGAG-3' and 5' ${ }^{\prime}$-TGGCTCTCATGTTTCCAGTG-3'; human CCND1, 5'-TCCTCTCCAAAATGCCAGAG -3' and $5^{\prime}$-GGCGGATTGG AAATGAACTT-3'; human CD CA2, 5' - ATGACAGACTTGACCAGAAAGGA-3' and 5'- CCGACGTTTGGAGGACAACA-3'; human LTBP1, $5^{\prime}$-GCCCTAATGGTGAGTGTTTGA-3' and $5^{\prime}$ - AGATCACAGGGGGATCAGG-3'; human LTBP2, 5' - TGCCC TAGTGGAAAAGGCTA- $3^{\prime}$ and $5^{\prime}$-TCACACACTCATC CGCATCT-3'; human KDM3A, 5'-GAGTTCAAGGCTG GGCTATTGT- $3^{\prime}$ and $5^{\prime}$-TTCAGCCACTTTGATGCAG CTA-3'; mouse Brg1, 5' -GCACCAAAATCAACGGGAC$3^{\prime}$ and 5'-CTAGGACCCAGCATTG CAC-3'; mouse Ccnb1, 5'-TGCAAACTGTAAGGTTGAAAGC-3' and 5'-TGTAGAGAGCCAAGTGGAAGG-3'; mouse Ltbp2, $5^{\prime \prime}$-AAACCCCTCAGCGACCCGCGGCTGC-3' and $5^{\prime \prime}$-T GCTTCTGTGAGGACCGGGTGCTCT-3'; mouse Kdm3a, 5'-AAATACGGTTTCGGGATG-3' and 5'-TACGGGTT TCTCGCTTCT- $3^{\prime}$. Data were analyzed by the $\Delta \Delta C T$ method and normalized to $18 \mathrm{~s}$ rRNA levels. All experiments were performed in triplicate wells and repeated three times.

\section{Scratch-wound healing/migration assay}

Cells were re-suspended in serum-free media. When the cells reached confluence, scratch wound was created by using a sterile micropipette tip. Cell migration was measured $24 \mathrm{~h}$ after the creation of the wound and calculated by Image Pro. Data were expressed as percentage migration compared to control arbitrarily set as 1 .

\section{MTT assay}

Cell proliferation was measured using an MTT kit (Abcam) per vendor's recommendation. Briefly, cells were plated in 12-well plates and allowed to attach overnight before exposure to $1 \% \mathrm{O}_{2}$ for $24 \mathrm{~h}$. MTT colorimetry was measured before $(0 \mathrm{~h})$ and after $(24 \mathrm{~h})$ the exposure. Data were expressed as percentage proliferation compared to control arbitrarily set as 1 .

\section{Chromatin Immunoprecipitation}

Chromatin immunoprecipitation (ChIP) assays were performed essentially as described before ${ }^{43,51-60}$. In brief, chromatin in control and treated cells were cross-linked with $1 \%$ formaldehyde. Cells were incubated in lysis buffer (150 mM NaCl, $25 \mathrm{mM}$ Tris pH 7.5, 1\% Triton X-100, $0.1 \%$ SDS, $0.5 \%$ deoxycholate) supplemented with protease inhibitor tablet and PMSF. DNA was fragmented into $~ 500$ bp pieces using a Branson 250 sonicator. Aliquots of lysates containing $200 \mu \mathrm{g}$ of protein were used for each immunoprecipitation reaction with anti-BRG1 (Abcam, ab110641), anti-KDM3A (Bethyl Laboratories, A301-538A), anti-trimethyl H3K4 (Millipore, 07-449), anti-dimethyl H3K9 (Millipore, 07-441), anti-acetyl H3 (Millipore, 06-599), anti-E2F1 (Santa Cruz, sc-193), antiSp1 (Santa Cruz, sc-14027), anti-HIF-1a (Santa Cruz, sc10790), or pre-immune IgG. For re-ChIP, immune complexes were eluted with the elution buffer (1\% SDS, $100 \mathrm{mM} \mathrm{NaCO}$ ), diluted with the re-ChIP buffer (1\% Triton X-100, $2 \mathrm{mM}$ EDTA, $150 \mathrm{mM} \mathrm{NaCl}, 20 \mathrm{mM}$ Tris $\mathrm{pH}$ 8.1), and subject to immunoprecipitation with a second antibody of interest. Precipitated genomic DNA was amplified by real-time PCR with the following primers: mouse Ccnb1 promoter, 5'-TAAACCTAAGCCCGGC AGAC-3' and 5'-CCCGATTCGAGAAGACACC-3'; mouse Ltbp2, 5'-ACGCATAACCTCATTCATGTACCG ATC-3' and 5'-AAAGCAATTCTCTAAATTCATA G-3'; mouse Kdm3a promoter, 5'-TCTGTTCCACAAGC ATTGACTGG-3' and 5'-AGGGCTATCACTATTGACA CCTGC-3'; mouse Gapdh promoter, 5'-ATCACTGCCACCCAGAAGACTGTGGA- $3^{\prime}$ and $5^{\prime}$-CTCATACCAG GAAATGAGCTTGACAAA- $3^{\prime}$. A total of $10 \%$ of the starting material is also included as the input. Data are then normalized to the input and expressed as \% recovery relative the input. All experiments were performed in triplicate wells and repeated three times.

\section{Statistical analysis}

Sample sizes reflected the minimal number needed for statistical significance based on power analysis and prior experience. Two-tailed Student $t$ test (for comparison of two groups) or one-way ANOVA with post-hoc Scheffe (for comparison of three or more groups) analyses were performed using an SPSS package. Unless otherwise specified, $P$ values $<0.05$ were considered statistically significant.

\section{Acknowledgements \\ This work was supported in part by grants from the National Natural Science Foundation of China (81770286, 81570420, and 81870326). \\ Author details \\ ${ }^{1}$ Key Laboratory of Targeted Intervention of Cardiovascular Disease and Collaborative Innovation Center for Cardiovascular Translational Medicine, Department of Pathophysiology, Nanjing Medical University, Nanjing, China. ${ }^{2}$ Department of Respiratory Medicine, The Affiliated Hospital of Nanjing University of Chinese Medicine, Jiangsu Province Hospital of Traditional Chinese Medicine, Nanjing, China. ${ }^{3}$ Department of Clinical Medicine and Laboratory Center for Experimental Medicine, Jiangsu Health Vocational College, Nanjing, China. ${ }^{4}$ Institute of Biomedical Research, Liaocheng University, Liaocheng, China}

\section{Author contributions}

Y.X. and J.X. conceived the project; Z.L., M.F., and Y.X. designed experiments; Z.L. and M.F. performed experiments and collected and analyzed data; Y.X. wrote the manuscript; J.X. provided funding and supervision. 


\section{Conflict of interest}

The authors declare that they have no conflict of interest.

\section{Publisher's note}

Springer Nature remains neutral with regard to jurisdictional claims in published maps and institutional affiliations.

Supplementary Information accompanies this paper at (https://doi.org/ 10.1038/s41389-019-0174-7)

Received: 5 July 2019 Revised: 14 October 2019 Accepted: 15 October 2019 Published online: 06 November 2019

\section{References}

1. Kehl, K. L., Lathan, C. S., Johnson, B. E. \& Schrag, D. Race, poverty, and initia implementation of precision medicine for lung cancer. J. Natl Cancer Inst. 111, 431-434 (2019).

2. Stram D. O., et al. Racial/ethnic differences in lung cancer incidence in the multiethnic cohort study: an update. J. Natl Cancer Inst. (2019).

3. Salgia, R. MET in lung cancer: biomarker selection based on scientific rationale. Mol. Cancer Ther. 16, 555-565 (2017).

4. Salem A., et al. Targeting hypoxia to improve non-small cell lung cancer outcome. J. Nat/ Cancer Inst. 110 (2018). https://doi.org/10.1093/jnci/djx160.

5. Swinson, D. E. et al. Hypoxia-inducible factor-1 alpha in non small cell lung cancer: relation to growth factor, protease and apoptosis pathways. Int. J. Cancer 111, 43-50 (2004).

6. Wang, W. et al. Diversity and specialization of mammalian SWI/SNF complexes. Genes Dev. 10, 2117-2130 (1996).

7. Hargreaves, D. C. \& Crabtree, G. R. ATP-dependent chromatin remodeling: genetics, genomics and mechanisms. Cell Res. 21, 396-420 (2011).

8. Bultman, S. et al. A Brg1 null mutation in the mouse reveals functional differences among mammalian SWI/SNF complexes. Mol. Cell 6, 1287-1295 (2000).

9. Fang, F. et al. Proinflammatory stimuli engage Brahma related gene 1 and Brahma in endothelial injury. Circulation Res. 113, 986-996 (2013).

10. Chen, D. et al. Brahma-related gene 1 (Brg1) epigenetically regulates CAM activation during hypoxic pulmonary hypertension. Cardiovascular Res. 100, 363-373 (2013).

11. Weng, X. et al. A crosstalk between chromatin remodeling and histone H3K4 methyltransferase complexes in endothelial cells regulates angiotensin IIinduced cardiac hypertrophy. J. Mol. Cell. Cardiol. 82, 48-58 (2015).

12. Li, N. et al. Brg1 regulates pro-lipogenic transcription by modulating SREBP activity in hepatocytes. Biochim. Biophys. Acta Mol. Basis Dis. 1864, 2881-2889 (2018).

13. Liu, L. et al. A cross talk between BRG1 and males absent on the first contributes to reactive oxygen species production in a mouse model of nonalcoholic steatohepatitis. Antioxid. Redox Signal. 30, 1539-1552 (2019).

14. Zhang, X. et al. Brg1 trans-activates endothelium-derived colony stimulating factor to promote calcium chloride induced abdominal aortic aneurysm in mice. J. Mol. Cell. Cardiol. 125, 6-17 (2018).

15. Medina, P. P. et al. Frequent BRG1/SMARCA4-inactivating mutations in human lung cancer cell lines. Hum. Mutat. 29, 617-622 (2008).

16. Orvis, $T$. et al. BRG1/SMARCA4 inactivation promotes non-small cell lung cancer aggressiveness by altering chromatin organization. Cancer Res. 74, 6486-6498 (2014).

17. Xue, Y. et al. SMARCA4 loss is synthetic lethal with CDK4/6 inhibition in nonsmall cell lung cancer. Nat. Commun. 10, 557 (2019).

18. Tagal, $\mathrm{V}$. et al. SMARCA4-inactivating mutations increase sensitivity to Aurora kinase A inhibitor VX-680 in non-small cell lung cancers. Nat. Commun. 8, 14098 (2017).

19. Ding, G. et al. MiR-199a suppresses the hypoxia-induced proliferation of nonsmall cell lung cancer cells through targeting HIF1alpha. Mol. Cell. Biochem. 384, 173-180 (2013).

20. Hou, S. Y. et al. Aquaporin-3 inhibition reduces the growth of NSCLC cells induced by hypoxia. Cell. Physiol. Biochem. 38, 129-140 (2016).
21. Beyer, S., Kristensen, M. M., Jensen, K. S., Johansen, J. V. \& Staller, P. The histone demethylases JMJD1A and JMJD2B are transcriptional targets of hypoxiainducible factor HIF. J. Biol. Chem. 283, 36542-36552 (2008).

22. Baylin, S. B. \& Jones, P. A. A decade of exploring the cancer epigenome biological and translational implications. Nat. Rev. Cancer 11, 726-734 (2011).

23. Murtha, M. \& Esteller, M. Extraordinary cancer epigenomics: thinking outside the classical coding and promoter box. Trends Cancer 2, 572-584 (2016).

24. Hohmann, A. F. \& Vakoc, C. R. A rationale to target the SWI/SNF complex for cancer therapy. Trends Genet. 30, 356-363 (2014).

25. Liu, K., Luo, Y., Lin, F. T. \& Lin, W. C. TopBP1 recruits Brg1/Brm to repress E2F1induced apoptosis, a novel pRb-independent and E2F1-specific control for cell survival. Genes Dev. 18, 673-686 (2004).

26. $\mathrm{Yeo}, \mathrm{H}$. et al. FoxO3 coordinates metabolic pathways to maintain redox balance in neural stem cells. EMBO J. 32, 2589-2602 (2013).

27. Zhang, B., Chambers, K. J., Faller, D. V. \& Wang, S. Reprogramming of the SWI/ SNF complex for co-activation or co-repression in prohibitin-mediated estrogen receptor regulation. Oncogene 26, 7153-7157 (2007).

28. Chuang, Y. S. et al. Promyelocytic leukemia protein in retinoic acid-induced chromatin remodeling of Oct4 gene promoter. Stem Cells 29, 660-669 (2011).

29. Deng, $X$. et al. Protein arginine methyltransferase 5 functions as an epigenetic activator of the androgen receptor to promote prostate cancer cell growth. Oncogene 36, 1223-1231 (2017).

30. Zhang, Y., Sif, S. \& DeWille, J. The mouse C/EBPdelta gene promoter is regulated by STAT3 and Sp1 transcriptional activators, chromatin remodeling and c-Myc repression. J. Cell. Biochem. 102, 1256-1270 (2007).

31. Kenneth, N. S., Mudie, S., van Uden, P. \& Rocha, S. SWI/SNF regulates the cellular response to hypoxia. J. Biol. Chem. 284, 4123-4131 (2009).

32. Sena, J. A., Wang, L. \& Hu, C. J. BRG1 and BRM chromatin-remodeling complexes regulate the hypoxia response by acting as coactivators for a subset of hypoxia-inducible transcription factor target genes. Mol. Cell. Biol. 33, 3849-3863 (2013).

33. Peng, $\mathrm{K}$. et al. Histone demethylase JMJD1A promotes colorectal cancer growth and metastasis by enhancing Wnt/beta-catenin signaling. J. Biol. Chem. 293, 10606-10619 (2018).

34. Fan, L. et al. Histone demethylase JMJD1A promotes alternative splicing of AR variant 7 (AR-V7) in prostate cancer cells. Proc. Natl Acad. Sci. USA 115, E4584-E4593 (2018).

35. Krieg, A. J. et al. Regulation of the histone demethylase JMJD1A by hypoxiainducible factor 1 alpha enhances hypoxic gene expression and tumor growth. Mol. Cell. Biol. 30, 344-353 (2010).

36. Ramadoss, S., Guo, G. \& Wang, C. Y. Lysine demethylase KDM3A regulates breast cancer cell invasion and apoptosis by targeting histone and the nonhistone protein p53. Oncogene 36, 47-59 (2017).

37. Wu Q., et al. The BRG1 chromatin remodeling enzyme links cancer cell metabolism and proliferation. Oncotarget 7, 38270-38281 (2016).

38. Sun, L. et al. A SUMOylation-dependent pathway regulates SIRT1 transcription and lung cancer metastasis. J. Natl Cancer Inst. 105, 887-898 (2013).

39. Cheng, $X$. et al. MKL1 potentiates lung cancer cell migration and invasion by epigenetically activating MMP9 transcription. Oncogene 34, 5570-5581 (2015).

40. Li, Y. et al. Regulation of a novel androgen receptor target gene, the cyclin B1 gene, through androgen-dependent E2F family member switching. Mol. Cell. Biol. 32, 2454-2466 (2012).

41. Li, Z. et al. Epigenetic activation of PERP transcription by MKL1 contributes to ROS-induced apoptosis in skeletal muscle cells. Biochim. Biophysica Acta Gene Regul. Mech. 1861, 905-915 (2018).

42. Li, Z. et al. BRG1 regulates NOX gene transcription in endothelial cells and contributes to cardiac ischemia-reperfusion injury. Biochim. Biophys. Acta Mol. Basis Dis. 1864, 3477-3486 (2018).

43. Shao, J. et al. Angiotensin II induced CSF1 transcription is mediated by a crosstalk between different epigenetic factors in vascular endothelial cells. Biochim. Biophys. Acta Gene Regul. Mech. 1862, 1-11 (2019).

44. Li, M. et al. Hepatic stellate cell-specific deletion of SIRT1 exacerbates liver fibrosis in mice. Biochim. Biophys. Acta 1863, 3202-3211 (2017).

45. Li, Z. et al. The histone methyltransferase SEID1A regulates thrombomodulin transcription in vascular endothelial cells. Biochim. Biophys. Acta Gene Regul. Mech. 1861, 752-761 (2018).

46. Yu, L., Li, Z., Fang, M. \& Xu, Y. Acetylation of MKL1 by PCAF regulates proinflammatory transcription. Biochim. Biophys. Acta Gene Regul. Mech. 1860, 839-847 (2017) 
47. Yang, Y. et al. Tanshindiol C inhibits oxidized low-density lipoprotein induced macrophage foam cell formation via a peroxiredoxin 1 dependent pathway. Biochim. Biophys. Acta Mol. Basis Dis. 1864, 882-890 (2018).

48. Zeng, S. et al. Hypermethylated in cancer 1 ( $\mathrm{HIC} 1)$ mediates high glucose induced ROS accumulation in renal tubular epithelial cells by epigenetically repressing SIRT1 transcription. Biochim. Biophys. Acta Gene Regul. Mech. 1861 917-927 (2018).

49. Fan, Z. et al. HIF-1alpha coordinates epigenetic activation of SIAH1 in hepatocytes in response to nutritional stress. Biochim. Biophys. Acta Gene Regul. Mech. 1860, 1037-1046 (2017).

50. Liu, L. et al. Myocardin-related transcription factor A (MRTF-A) contributes to acute kidney injury by regulating macrophage ROS production. Biochim. Biophys. Acta Mol. Basis Dis. 1864, 3109-3121 (2018).

51. Fan, Z. et al. An interaction between MKL1, BRG1, and C/EBPbeta mediates palmitate induced CRP transcription in hepatocytes. Biochim. Biophys. Acta Gene Regul. Mech. 1862, 194412 (2019).

52. Kong, M. et al. Serum response factor (SRF) promotes ROS generation and hepatic stellate cell activation by epigenetically stimulating NCF1/2 transcription. Redox Biol. 26, 101302 (2019).

53. Kong M., et al. Ablation of serum response factor in hepatic stellate cells attenuates liver fibrosis. J. Mol. Med. (Berl) (2019).
54. Li, N. et al. The chromatin remodeling protein BRG1 regulates APAP-induced liver injury by modulating CYP3A11 transcription in hepatocyte. Biochimica et. biophysica acta Mol. basis Dis. 1864, 3487-3495 (2018).

55. Li, N. et al. Brahma related gene 1 (Brg1) contributes to liver regeneration by epigenetically activating the Wnt/beta-catenin pathway in mice. FASEB J. 33 327-338 (2019).

56. Li, Z. et al. A non-autonomous role of MKL1 in the activation of hepatic stellate cells. Biochim. Biophys. Acta Gene Regul. Mech. 1862, 609-618 (2019).

57. Liu, L. et al. BRG1 regulates endothelial-derived IL-33 to promote ischemiareperfusion induced renal injury and fibrosis in mice. Biochim. Biophys. Acta Mol. Basis Dis. 1865, 2551-2561 (2019).

58. Weng, $X$. et al. Class II transactivator (CIITA) mediates IFN-gamma induced eNOS repression by enlisting SUV39H1. Biochim. Biophys. Acta Gene Regul. Mech. 1862, 163-172 (2019).

59. Yang, Y., Liu, L., Fang, M., Bai, H. \& Xu, Y. The chromatin remodeling protein BRM regulates the transcription of tight junction proteins: implication in breast cancer metastasis. Biochim. Biophys. Acta Gene Regul. Mech. 1862, 547-556 (2019).

60. Yang, Y. et al. The chromatin remodeling protein BRG1 links ELOVL3 transactivation to prostate cancer metastasis. Biochim. Biophys. Acta Gene Regul. Mech. 1862, 834-845 (2019). 